\title{
Improving CAR T cell immunotherapy-mediated remissions for pediatric leukemia
}

\author{
David M. Barrett
}

Center for Childhood Cancer Research Cell Therapy Program, Children's Hospital of Philadelphia, Philadelphia, Pennsylvania, USA.

\begin{abstract}
Chimeric antigen receptor (CAR) T cells are an effective therapy for relapsed or refractory pediatric B cell leukemia. Analysis of the starting material, the T cells collected from the patient prior to CAR manufacture, reveals possible biomarkers of cells destined to perform poorly in patients. Long-term followup shows that long periods of B cell aplasia, a marker of in vivo CAR activity, are associated with longer remission but also a higher chance of antigennegative relapse. The role of transplantation as consolidative therapy is unclear in this nonrandomized data, but clearly warrants further study.
\end{abstract}

\section{Will CAR T cells last for my patient?}

A highly anticipated long-term follow-up of pediatric acute lymphoblastic leukemia (ALL) patients treated with a chimeric antigen receptor (CAR) targeting CD19, the report from Finney et al., takes a few steps toward answering this question (1). Initially reported in 2017, the NCT02028455 trial had the same exciting and tantalizing high early remission rates of trials at other centers, including the National Institutes of Health and Children's Hospital of Philadelphia (2-4). This was highly encouraging, since each trial had different ways to make a CAR $\mathrm{T}$ cell product and different patient characteristics, giving hope that CAR T cells were a generalizable strategy for refractory leukemia.

However, as most pediatric oncologists know, getting a patient into remission is only the start when it comes to leukemia. Questions abound: how long will a patient stay in remission, and what additional therapy (if any) is needed to stay there? CAR T cells add a new dimension to these questions, as we must consider the particular features of $\mathrm{T}$ cells along with the leukemia-intrinsic factors to decide on the need for post-CAR consolidative therapy, typically allogeneic hematopoietic stem cell transplantation (Figure 1).

\section{Observations from the current study}

Finney et al. report that 5 of the 43 patients from the study experienced either no response or relapsed very quickly (within 63 days), and had an $88.3 \%$ complete response rate at 63 days. Characteristics of the CAR T cell starting material indicate that patients with a dysfunctional response may have had $\mathrm{T}$ cells poorly suited to CAR $\mathrm{T}$ cell manufacture, with high expression of the exhaustion marker LAG-3 in the $\mathrm{CD}^{+}$cells. This fits with a growing body of research indicating that the quality of the $\mathrm{T}$ cells harvested from the patients is a major factor in how those cells will ultimately perform as CAR T cells (5-9). This also opens the door for adapting manufacturing support to each patient's T cell needs, further personalizing an already highly patient-specific therapy.

Finney et al. used a manufacturing strategy that splits $\mathrm{CD} 4^{+}$and $\mathrm{CD} 8^{+} \mathrm{T}$ cells, grows them in different conditions, and then mixes them back together in a 1:1 ratio

Related Article: p. 2123

Conflict of interest: The author has declared that no conflict of interest exists.

using a surface selection marker. This is in contrast to the product now known as tisagenlecleucel (Kymriah), developed at the University of Pennsylvania in partnership with Novartis, where $\mathrm{T}$ cells are not separated, but $\mathrm{CD}^{+}$and $\mathrm{CD} 8^{+} \mathrm{T}$ cells are grown together in the same cytokine milieu (10). Both products have fabulous initial results, indicating that there are potentially many possible ways to manufacture CAR T cells. Do the positive results provide clues as to what could have been done differently (if anything) during ex vivo manufacture for the $\mathrm{T}$ cells from those five patients to make them effective? Are cells damaged by prior therapy unsalvageable? Is a post-infusion booster strategy ( $\mathrm{T}$ cell antigen presenting cells), such as the one the authors propose, a way to manufacture CAR T cells?

\section{Could universal CAR T cells be} the answer?

Some trials require fresh pheresis for manufacturing, while others accept prior frozen products. If a patient is identified as needing CAR $\mathrm{T}$ cells, but their current lymphocytes are too damaged from chemotherapy, what does one do? Perhaps we will see something akin to cord blood banking, with families and patients partnering with centers to bank healthy $\mathrm{T}$ cells "just in case." More narrowly, what about banking $\mathrm{T}$ cells at diagnosis for high-risk patients? There would unquestionably be a number of products that are stored and never used. How many unused products is it acceptable to store for the one child who can be treated that otherwise could not?

This leads to another important question in the field: if the $\mathrm{T}$ cells from cancer patients are intrinsically bad (whether from the tumor or the result of therapy), why not use off-the-shelf CAR $\mathrm{T}$ cells from healthy donors? So-called universal CAR $\mathrm{T}$ cells are attractive in many ways. They are immediately available, made from healthy $\mathrm{T}$ cells that have never seen chemotherapy, and potentially less expensive. An early report of 


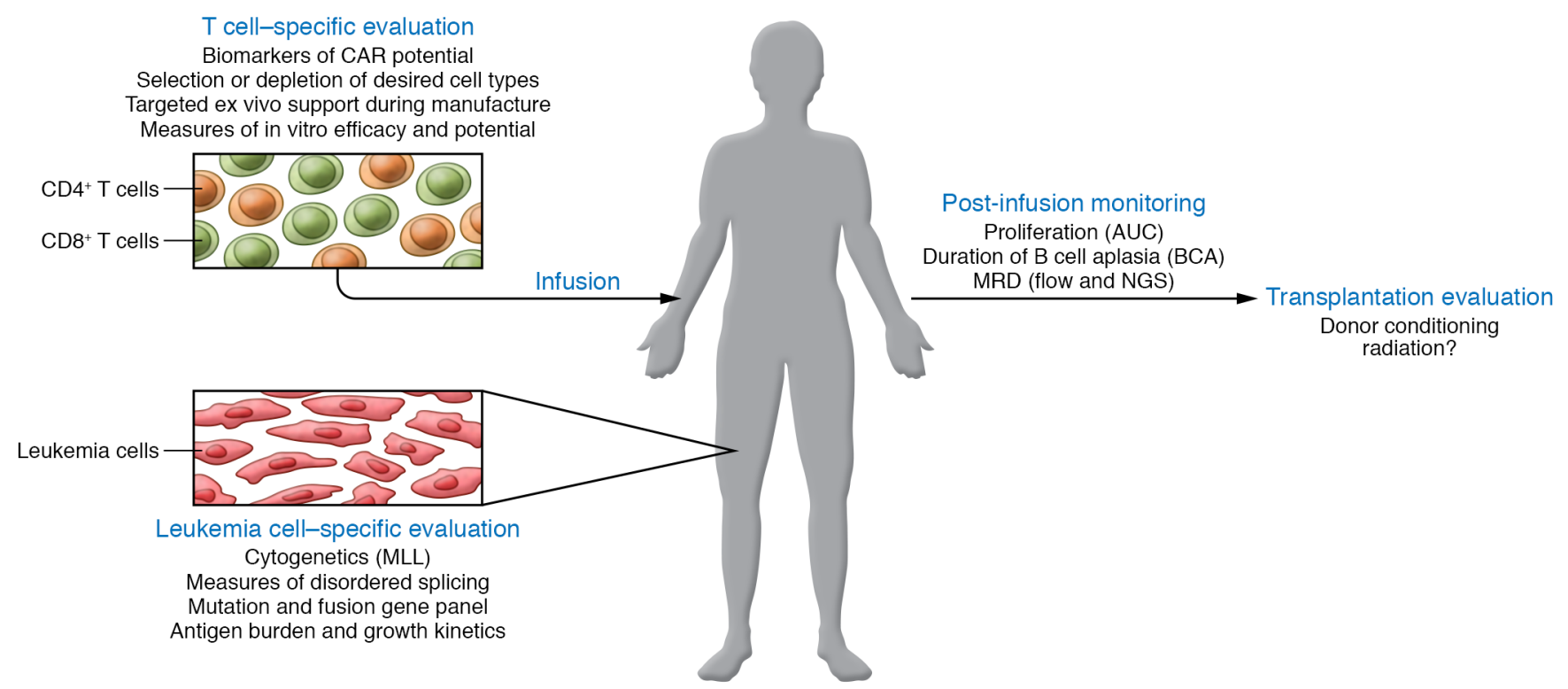

Figure 1. Model of the factors influencing CAR T cell response and persistence. T cell intrinsic factors including the CD4/CD8 ratio, memory phenotype, expression of exhaustion markers, and metabolic profile can inform how the cells are supported in the lab during CAR manufacture. Standard assessments of CAR T cell function such as cytokine release and polyfunctional index will be important for comparison across trials. Leukemia intrinsic factors such as driver lesion (cytogenetic, fusion, or mutation), preexisting antigen-low clones, and overall antigen burden will inform relapse risk. After infusion, patient monitoring with CAR T cell proliferation area under the curve (AUC), duration of BCA, and assessment with ultrasensitive next-generation sequencing (NCS) MRD will inform the ultimate decision for post-CAR consolidation, such as use of allogeneic transplantation.

two patients treated with universal CAR $\mathrm{T}$ cells showed many of the challenges found with this kind of product, including the importance of purity, the potential for severe toxicity, possible weaknesses in safety systems, and the need for consolidative therapy with transplantation (11). Universal CAR $\mathrm{T}$ cells are expected to have short persistence from eventual immunologic rejection, and the degree of complexity of genetic manipulation to overcome this is quite high. What is the cost of making universal CAR $\mathrm{T}$ cells once all the genetic manipulation and possible need for multiple infusions is accounted for, especially if the cells do not achieve lasting remission and require consolidative transplantation? Given the high interest in this technology, only time and well-designed trials will answer these questions.

\section{Antigen escape}

A pressing question is what to do after a patient achieves remission with CAR $\mathrm{T}$ cells. Finney et al. report that 13 of 38 patients went on to undergo consolidative transplantation, with 10 patients alive and in remission at the time of report and 3 relapses (one of which was antigen negative). Twenty of the 25 patients who got no further therapy experienced a relapse, half of them antigen negative. Looking at the individual data, it is striking that one patient (S11) had an antigen-negative relapse at 38 months. That patient had B cell aplasia (BCA) for 38 months as well, usually a sign that CAR $\mathrm{T}$ cells are still active. Finney et al. report that long BCA ( $>6$ months) is positively correlated with extended remission but also correlated with more antigennegative relapse. These are not randomized data, however, and we must be cautious about how we interpret these results with regard to transplantation.

The leukemia intrinsic factors intersect with the CAR T cell power, as we currently lack any ability to know which leukemias will ultimately go on to antigen escape. We also do not truly know if transplantation will suppress those events, or if there is a window in which transplantation will work, but outside of which it will not. Minimal residual disease (MRD) testing has redefined treatment of pediatric leukemia because it accurately reflects how well a patient is responding to treatment at previously unreachable levels of detection (12). All we know about MRD, however, is based on chemotherapy-induced MRD states, not immunologically induced ones. Is an MRD-negative result from an immunotherapy as prognostic as one induced by chemotherapy? What about a nextgeneration sequencing MRD result that is several logs more sensitive than a flow cytometry-based result (13)? We will need to integrate this kind of response data into the $\mathrm{T}$ cell expansion and BCA data to get a full picture of relapse risk. If antigennegative relapse is possible after three years, then a long follow-up is required before we will truly know the answers to the aforementioned questions.

Finney et al. also address the suppression of antigen escape, describing the common strategy of adding another target antigen (CD22) to the CAR T cell pool (14). Although there is hope for this strategy, leukemia has already figured out an escape plan for that as well - lineage switch. Some B cell leukemias can switch lineages, activating myeloid expression markers and suppressing lymphoid ones (including CD22) and this has been reported in CAR T cell therapy $(15,16)$. Can we predict lineage switch and pre-empt it with transplantation, and will transplantation actually work for that purpose? Detailed translational science can answer this, now that we have clinical samples to study. 


\section{Hope for patients \\ who underwent prior transplantation}

As we evaluate responses and worry over transplantation, another question surfaces: what about patients who already underwent transplantation? Their T cells will be from the donor stem cells and have potentially different chemotherapy exposure and repertoires than those patients who have been receiving up-front intensive therapy. Does that matter? Most of the patients reported by Maude et al. underwent a prior transplantation, and certainly the initial responses were excellent (4). But patients undergoing a second allogeneic transplantation have a high chance of transplant-related mortality and historically a high rate of relapse, though not in the context of post-CAR T cell remission (17). We must factor all this in to our evaluation of BCA, MRD, and other biomarkers as we weigh risks and benefits for each patient.

In summary, the report from Finney et al. provides a long-term look at outcomes from patients treated with CAR $\mathrm{T}$ cells and some clues as to what $\mathrm{T}$ cell factors are associated with response and durable remission. We have new hints at the answers to key questions in the field, but still no final way to answer the one question every family has: can CAR T cells cure my child's leukemia?
Address correspondence to: David M. Barrett, CCCR Cell Therapy Program, Children's Hospital of Philadelphia, Oncology/BMT CTRB 3032, 3501 Civic Center Boulevard, Philadelphia, Pennsylvania 19104-4318, USA. Phone: 267.426.9740; Email: barrettd@email.chop.edu.

1. Finney OC, et al. CD19 CAR T cell product and disease attributes predict leukemia remission durability. J Clin Invest. 2019;129(5):2123-2132.

2. Gardner RA, et al. Intent-to-treat leukemia remission by CD19 CAR T cells of defined formulation and dose in children and young adults. Blood. 2017;129(25):3322-3331.

3. Lee DW, et al. T cells expressing CD19 chimeric antigen receptors for acute lymphoblastic leukaemia in children and young adults: a phase 1 dose-escalation trial. Lancet. 2015;385(9967):517-528.

4. Maude SL, et al. Chimeric antigen receptor $\mathrm{T}$ cells for sustained remissions in leukemia. N Engl J Med. 2014;371(16):1507-1517.

5. Berger C, Jensen MC, Lansdorp PM, Gough M, Elliott C, Riddell SR. Adoptive transfer of effector $\mathrm{CD}^{+} \mathrm{T}$ cells derived from central memory cells establishes persistent $\mathrm{T}$ cell memory in primates. J Clin Invest. 2008;118(1):294-305.

6. Das RK, Vernau L, Grupp SA, Barrett DM. Naïve T-cell deficits at diagnosis and after chemotherapy impair cell therapy potential in pediatric cancers [published online ahead of print January 10, 2019]. Cancer Discov. https://doi. org/10.1158/2159-8290.CD-18-1314.

7. Fraietta JA, et al. Ibrutinib enhances chimeric antigen receptor T-cell engraftment and efficacy in leukemia. Blood. 2016;127(9):1117-1127.

8. Fraietta JA, et al. Determinants of response and resistance to $\mathrm{CD} 19$ chimeric antigen receptor
(CAR) T cell therapy of chronic lymphocytic leukemia. Nat Med. 2018;24(5):563-571.

9. Singh N, Perazzelli J, Grupp SA, Barrett DM. Early memory phenotypes drive $\mathrm{T}$ cell proliferation in patients with pediatric malignancies. Sci Transl Med. 2016;8(320):320ra3.

10. Maude SL, et al. Tisagenlecleucel in children and young adults with B-cell lymphoblastic leukemia. N Engl J Med. 2018;378(5):439-448.

11. Qasim W, et al. Molecular remission of infant B-ALL after infusion of universal TALEN gene-edited CAR T cells. Sci Transl Med. 2017;9(374):eaaj2013.

12. Borowitz MJ, et al. Clinical significance of minimal residual disease in childhood acute lymphoblastic leukemia and its relationship to other prognostic factors: a Children's Oncology Group study. Blood. 2008;111(12):5477-5485.

13. Hay KA, et al. Factors associated with durable EFS in adult B-cell ALL patients achieving MRD-negative CR after CD19 CAR-T cells [published online ahead of print February 6, 2019]. Blood.https:// doi.org/10.1182/blood-2018-11-883710.

14. Fry TJ, et al. CD22-targeted CAR T cells induce remission in B-ALL that is naive or resistant to CD19-targeted CAR immunotherapy. Nat Med. 2018;24(1):20-28.

15. Jacoby E, et al. CD19 CAR immune pressure induces B-precursor acute lymphoblastic leukaemia lineage switch exposing inherent leukaemic plasticity. Nat Commun. 2016;7:12320.

16. Oberley MJ, et al. Myeloid lineage switch following chimeric antigen receptor $\mathrm{T}$-cell therapy in a patient with TCF3-ZNF384 fusion-positive B-lymphoblastic leukemia. Pediatr Blood Cancer. 2018;65(9):e27265.

17. Naik S, et al. Outcomes after second hematopoietic stem cell transplantations in pediatric patients with relapsed hematological malignancies. Biol Blood Marrow Transplant. 2015;21(7):1266-1272 